\title{
Altered plasma glutamate and glutamine levels in patients with drug-resistant and drug-responsive symptomatic focal epilepsy
}

Wei Wang, PhD, Yanchuan Wu, MSc, Xiaoling Li, PhD, Lin Li, PhD, Ke Sun, MD, Suying Yan, MSc.

\footnotetext{
ABSTRACT

الأهداف : مقارنة مستويات الجلوتامات في البلازما والجلوت تامين

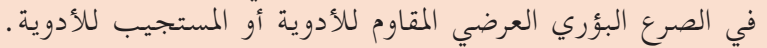

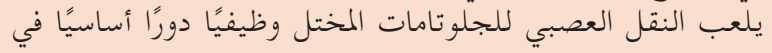

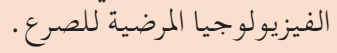

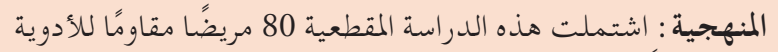

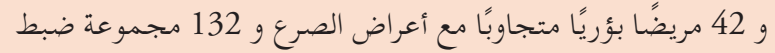

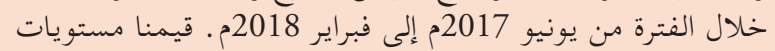

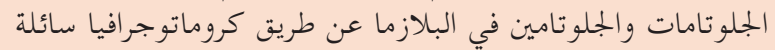

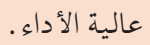

النتائج : المرضى الذين يعانون من الصرع البؤري المقاوم للأدوية

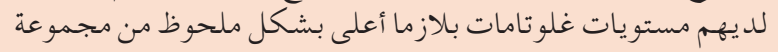

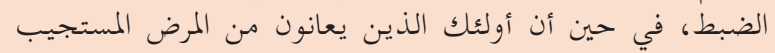

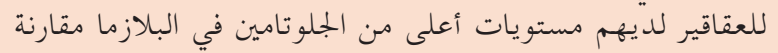

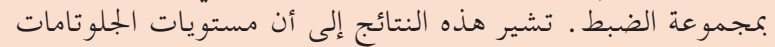

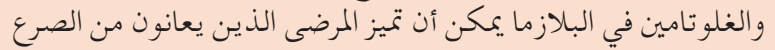

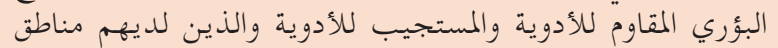

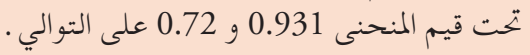

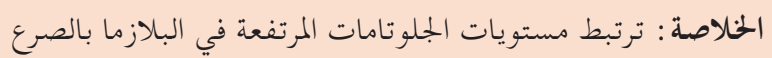

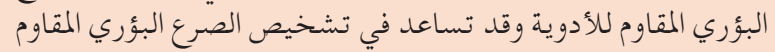

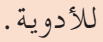

Objectives: To compare the levels of plasma glutamate and glutamine in drug-resistant or drug-responsive symptomatic focal epilepsy. Dysfunctional glutamate neurotransmission plays an essential role in epilepsy pathophysiology.

Methods: This cross-sectional study included 80 drug-resistant and 42 drug-responsive symptomatic focal epileptic patients and 132 healthy controls from June 2017 to February 2018. Plasma glutamate and glutamine levels were assessed via high-performance liquid chromatography.
}

Results: Patients with drug-resistant symptomatic focal epilepsy had significantly higher plasma glutamate levels than healthy controls, whereas those with the drug-responsive disease had higher plasma glutamine levels than healthy controls. These results indicate that plasma glutamate and glutamine levels can discriminate patients with drug-resistant and drug-responsive symptomatic focal epilepsy from control individuals with the areas under the curve values of 0.931 and 0.72 , respectively.

Conclusion: Elevated plasma glutamate levels are associated with drug-resistant symptomatic focal epilepsy and may aid the diagnosis of drug-resistant symptomatic focal epilepsy.

Neurosciences 2021; Vol. 26 (4):315-322 doi: 10.17712/nsj.2021.4.20210041

From the Department of Pharmacy (Wang, Li, Lin Li, Yan); from the Department of Central Laboratory (Wu); from the Department of Functional Neurosurgery (Sun); from the Beijing Institute of Functional Neurosurgery (Sun), Xuanwu Hospital, Capital Medical University, and from the Beijing Municipal Geriatric Medical Research Center (Wu), Beijing, China.

Received 12th April 2021. Accepted 22nd June 2021.

Address correspondence and reprint request to: Dr. Suying Yan, Department of Pharmacy, Xuanwu Hospital, Capital Medical University, Beijing, China. E-mail: yansuying10@sina.cn ORCID ID: https://orcid.org/0000-0001-7223-6467

$\mathrm{E}$ pilepsy, which affects more than 70 million people worldwide, is characterized by recurrent spontaneous seizures and associated with numerous neurological, cognitive, and psychosocial consequences. Although anti-epileptic drugs (AEDs) are the first line of treatment, these drugs are ineffective in approximately one-third of epilepsy patients who have what is referred to as drug-resistant epilepsy (DRE). ${ }^{1}$ Patients with 
DRE commonly have a diminished quality of life with inadequate employment and increased mortality rates.

As an excitatory neurotransmitter, glutamate plays a crucial role in neuronal plasticity and maintaining cognitive functions. In addition to glutamate, $\gamma$-aminobutyric acid (GABA) as an inhibitory neurotransmitter and the non-neuroactive precursor glutamine is also involved in glutamate/GABAglutamine cycling. ${ }^{2}$ Excessive levels of glutamate have been shown to be potently neurotoxic, leading to neuronal cell death and causing neuropsychiatric disorders, including epilepsy. ${ }^{3}$

Numerous microdialysis studies in the brains of patients with DRE have confirmed elevated extracellular glutamate levels in epileptogenic sites including the cortex and hippocampus. ${ }^{4-6}$ However, whether extracellular glutamate and glutamine levels are altered in the peripheral system of patients with DRE remains unknown. Blood tests represent a beneficial alternative to microdialysis due to their minimally invasive nature, straight forward implementation, and better adherence. Two studies have demonstrated that glutamate and glutamine levels in plasma are positively associated with those in cerebrospinal fluid. ${ }^{7,8}$ Therefore, we hypothesized that glutamate and glutamine alterations in plasma can be assumed to reflect changes in their levels in the brain. To identify a potential indicator that could aid the diagnosis of DRE, the present study compared plasma glutamate and glutamine levels between patients with drug-resistant or drug-responsive symptomatic focal epilepsy and healthy controls.

Methods. A total of 80 in-patients diagnosed with drug-resistant symptomatic focal epilepsy in the Functional Neurosurgical ward in Xuanwu hospital, Capital Medical university, Beijing, China, from June 2017 to February 2018 were enrolled in this study (DRE group). These patients were candidates for vagal nerve stimulation or epilepsy surgery. A total of 46 outpatients diagnosed with drug-responsive symptomatic focal epilepsy treated at the epilepsy clinic of our hospital within the same time frame also were included in this study (DSE group). A total of 132 healthy controls were

Disclosure. This study was funded by the National Natural Science Foundation of China (Grant No. 81503158), the Beijing Municipal Administration of Hospitals' Youth Program (Grant No. QML20160808) and the Beijing Municipal Administration of Hospitals' Ascent Plan (Grant No. DFL20190801), Beijing, China. recruited during health checkups at our hospital from June 2017 to February 2018. The study protocol was approved by the human ethical committee of Xuanwu hospital, and all participants signed an informed consent form. The study was conducted in compliance with the Declaration of Helsinki.

Drug-resistant symptomatic focal epilepsy was diagnosed according to the definition of DRE from the International league against epilepsy: sustained seizure freedom cannot be achieved with 2 tolerated appropriately chosen AEDs. ${ }^{9}$ Drug-responsive symptomatic focal epilepsy was diagnosed according to the definition of drug-responsive epilepsy (DSE): no seizures have occurred for at least one year prior to the last follow-up in patients with epilepsy treated with AEDs. ${ }^{10}$ Patients with tumors, hyperammonemia, or impaired liver or renal function were excluded from this study.

Measurement of plasma glutamate and glutamine levels. Whole blood samples were collected from the patients and healthy controls in the early morning after overnight fasting, while the patients were quietly resting. All samples were collected while the patients were taking prescribed AEDs. After centrifugation of venous blood samples, plasma samples were frozen and stored for later analysis of plasma glutamate and glutamine levels using reverse-phase high-performance liquid chromatography (RP-HPLC). The chemicals and reagents used in this study, except for methanol, were purchased from Sigma-Aldrich (St Louis, MO, USA). Methanol was supplied by Thermo Fisher Scientific (Waltham, MA, USA). High-performance liquid chromatography was carried out using an Agilent 1200 HPLC-fluorescence detector system (Agilent Technologies, Santa Clara, CA, USA) equipped with a G1321A fluorescence detector, G1322A on-line solvent degasser, G1311A pump, G1316A column oven, and G1329A auto-sampler. A Kromasil C18 column $(150 \times 4.6 \mathrm{~mm}, 5 \mu \mathrm{M}$; SigmaAldrich) was used for chromatographic separation. Gradient elution was formulated using a mobile phase composed of $100 \mathrm{mM}$ sodium acetate buffer (containing $0.05 \%$ tetrahydrofuran, $\mathrm{pH} 6.0, \mathrm{v} / \mathrm{v}$ ), (solvent $\mathrm{A}$ ) and methanol (solvent B). The gradient was programmed as follows: 65:35, solvent $A$ : solvent $B$ at 0 min and held for $7 \mathrm{~min}$; 40:60, solvent $A$ : solvent $B$ at $7 \mathrm{~min}$ and held for $9 \mathrm{~min}$; 95:5, and solvent A: solvent $B$ at $16 \mathrm{~min}$ and held for $4 \mathrm{~min}$. The flow rate was set to $1 \mathrm{ml} / \mathrm{min}$ with a column temperature at $30{ }^{\circ} \mathrm{C}$ and an injection volume of $5 \mu \mathrm{l}$. Fluorescence detection was carried out with an emission wave length of $450 \mathrm{~nm}$ and an excitation wave length of $340 \mathrm{~nm}$. Working standard solutions of glutamate and glutamine were prepared and used 
to generate calibration curves. For analysis, the stored plasma samples were thawed, mixed with methanol at a ratio of $1: 3$, and centrifuged at $12,000 \mathrm{rpm}$ for $20 \mathrm{~min}$ at $4{ }^{\circ} \mathrm{C}$. The supernatant was collected and derivatized with $25 \mathrm{mg}$ o-phthalaldehyde (OPA) dissolved in a mixture of $300 \mu \mathrm{l}$ methanol, $30 \mu \mathrm{l} \beta$-mercaptoethanol, and $4 \mathrm{ml}$ of $0.4 \mathrm{M}$ sodium borate buffer at $\mathrm{pH} 9.6$ before HPLC analysis. Chromatographic peaks were identified according to retention time in comparison with the retention times for the standard solutions. The levels of glutamate and glutamine in each sample were quantified relative to the peak areas of the corresponding substances.

Statistical analysis. The hypothesized normal distribution was tested with the Shapiro-Wilk test. Data was presented as the median (25\% percentile, $75 \%$ percentile). Differences between the healthy controls and patients with DRE or DSE were analyzed using $\times 2$ tests for qualitative variables and the Mann-Whitney $U$ test for quantitative variables. Differences in glutamate or glutamine levels between 2 groups (DRE vs. control, DSE vs. control) were assessed using analysis of covariance (ANCOVA) with age as a covariate. Univariate and multivariate binary logistic regression analyses were applied to determine the ability of plasma levels of glutamate and glutamine to distinguish DRE or DSE patients from the controls with consideration of gender, age, and body mass index (BMI). Receiver operating characteristic (ROC) curve analyses were used to measure the diagnostic performance of the plasma levels of glutamate and glutamine. Area under the ROC curve (AUC) values were calculated to determine the diagnostic accuracy of the plasma levels of glutamate and glutamine. The optimal cut-off values for plasma levels of glutamate and glutamine were determined using the Youden index method, based on the value at which the highest sum of specificity and sensitivity was achieved with equal weight. ${ }^{11}$ Associations between plasma glutamate levels in DRE patients and clinical parameters such as gender, age, BMI, disease duration, seizure frequency, and AED utilization profile at the last clinic visit were assessed with ordinal logistic regression analysis. The Statistical Package for the Social Science (SPSS) software version 25.0 (IBM Corp., Armonk, NY, USA) was used for data analysis, with a 2-sided $p$-value $<0.05$ indicating statistical significance.

Results. As shown in Table 1, at the last clinic visit, the patients in the DRE group were receiving either monotherapy consisting of carbamazepine (CBZ), oxcarbazepine (OXZ), phenytoin (PHT), lamotrigine (LTG), or levetiracetam (LEV) or different combinations of AEDs, including OXZ, CBZ, topiramate (TPM), valproate (VPA), LEV, LTG, phenobarbital (PHB), clonazepam (CZP), and PHT. At the last clinic visit, the patients in the DSE group were maintained either on monotherapy consisting of CBZ, OXZ or PHT or various combinations of AEDs involving the same polytherapy regimens as used for the patients in the DRE group, with the exception of CZP.

Since a considerable difference in age was found between the DRE patients and the controls and a marginal difference in age was detected between the DSE patients and the controls, ANCOVA was performed to adjust for this factor (Table 2). With adjustment for age, the glutamate level in the DRE patients remained significantly higher than that in the controls $(p<0.001)$. Consistently, the difference in glutamine levels between the DSE patients and the controls also remained significant after adjustment for age $(p<0.001)$.

Univariate logistic regression analysis demonstrated gender $(p=0.675)$, BMI $(p=0.786)$, and glutamine concentration $(p=0.176)$ were not associated with DRE and thus could not discriminate DRE patients from controls (Table 3).

The multivariate logistic regression model based on factors identified as significant by the univariate regression model $(p<0.05)$ revealed that only the plasma glutamate level was strongly and positively related to DRE and able to discriminate DRE patients from the controls.

Univariate logistic regression analysis revealed that gender $(p=0.83)$, age $(p=0.282)$, BMI $(p=0.769)$, and glutamate concentration $(p=0.083)$ were not associated with DSE and thus not able to discriminate DSE patients from controls (Table 4). However, the plasma glutamine level was positively correlated with DSE and able to discriminate DSE patients from controls (OR: 1.009 , 95\% CI: [1.005-1.012], $p<0.001)$.

For further validation, ROC curve analysis as shown in Figure 1 was performed to assess the ability of variable to discriminate between DRE patients and controls as well as between DSE patients and controls. A plasma glutamate concentration cut-off of $143.944 \mu \mathrm{M}$ accurately discriminated the DRE patients from the controls with $87.5 \%$ sensitivity and $87.9 \%$ specificity. A plasma glutamine concentration cut-off of 871.118 $\mu \mathrm{M}$ discriminated the DSE patients from the controls, but the sensitivity and specificity were $54.3 \%$ and $88.6 \%$, respectively. These findings suggest that the plasma glutamate concentration has a strong ability to discriminate between DRE patients and controls with high sensitivity and specificity. Additionally, the plasma glutamine concentration has a good ability to 
discriminate between DSE patients and controls, but with a relatively lower value of sensitivity plus specificity. The impacts of gender, age, BMI, duration of epilepsy, seizure frequency, and AED utilization profile at the last clinic visit on plasma glutamate concentrations in DRE patients were analyzed and shown in Table 5. Multivariate ordinal logistic regression analysis identified male sex and elevated BMI as positively associated with an increased plasma glutamate concentration.

Discussion. In this study, we performed HPLC analysis to evaluate differences in plasma glutamate and glutamine levels between patients with drug-resistant or drug-responsive symptomatic focal epilepsy and healthy controls. The results indicated that, compared with those among the normal controls, the plasma glutamate levels were markedly higher in the DRE patients and the plasma glutamine levels were considerably increased in the DSE patients. The plasma glutamate level showed significant potential for distinguishing between DRE patients and controls, with an AUC of 0.931 (95\% CI: [0.893-0.968]). A cut-off value of $143.944 \mu \mathrm{M}$ glutamate yielded a sensitivity of $87.5 \%$ and specificity of $87.9 \%$ for the detection of DRE patients. The plasma glutamine level also provided somewhat accurate discrimination of DSE patients from controls.

Alterations of plasma glutamate and glutamine levels in DRE patients. The results of the present study are consistent with the detection of elevated glutamate levels in a previous study that reported

Table 1 - Characteristics of the healthy control participants as well as the patients with drug-resistant epilepsy (DRE) and drug-responsive epilepsy (DSE).

\begin{tabular}{|c|c|c|c|c|c|}
\hline Characteristics & Healthy controls $(n=132)$ & DRE patients $(n=80)$ & $P$-value & DSE patients $(n=46)$ & $P$-value \\
\hline \multicolumn{6}{|l|}{ Gender } \\
\hline Male & 77 & 49 & 0.675 & 26 & 0.83 \\
\hline Female & 55 & 31 & & 20 & \\
\hline Age (years) & $29(26,34)$ & $27(23.25,33.75)$ & 0.009 & $27(22,34.25)$ & 0.056 \\
\hline $\mathrm{BMI}\left(\mathrm{kg} / \mathrm{m}^{2}\right)$ & $23.65(21.8,25.70)$ & $22.69(19.99,26.92)$ & 0.254 & $22.67(20.90,25.51)$ & 0.27 \\
\hline Duration of epilepsy (years) & N/A & $13(10,21.75)$ & $\mathrm{N} / \mathrm{A}$ & $9(4.75,20)$ & N/A \\
\hline \multicolumn{6}{|c|}{ Seizure frequency in the day prior to blood draw $n$ (\%) } \\
\hline Daily & N/A & $28(35)$ & N/A & N/A & N/A \\
\hline Weekly & N/A & $20(26)$ & N/A & $\mathrm{N} / \mathrm{A}$ & $\mathrm{N} / \mathrm{A}$ \\
\hline Monthly & N/A & $32(40)$ & N/A & N/A & N/A \\
\hline \multicolumn{6}{|c|}{ AED utilization profile at the last clinic visit n (\%) } \\
\hline Monotherapy & N/A & $13(16.3)$ & N/A & $16(34.8)$ & N/A \\
\hline Polytherapy & N/A & $67(83.8)$ & N/A & $30(65.2)$ & N/A \\
\hline Glutamate $(\mu \mathrm{M})$ & $111.358(99.088,131.814)$ & $187.68(152.505,215.515)$ & $<0.001$ & $115.074(91.71,152.072)$ & 0.523 \\
\hline Glutamine $(\mu \mathrm{M})$ & $762.65(709.775,820.926)$ & $784.142(726.821,864.685)$ & 0.111 & $880.132(760.29,954.172)$ & $<0.001$ \\
\hline
\end{tabular}

BMI - body mass index, AED - antiepileptic drug, N/A - not applicable. Data for age, BMI, duration of eplilepsy, and levels of glutamate and glutamine are presented as median ( $25 \%$ percentile, $75 \%$ percentile)

Table 2 - Plasma glutamate and glutamine levels in the DRE, DSE, and control groups (adjusted for age as a covariate)

\begin{tabular}{lcccc}
\hline Variables & Mean & $95 \%[\mathrm{CI}]$ & Test statistics & $P$-value \\
\hline $\begin{array}{l}\text { Glutamate }(\mu M) \\
\text { Control }\end{array}$ & 112.618 & {$[106.687-118.549]$} & & \\
$\quad$ DRE & 187.483 & {$[179.838-195.129]$} & $\mathrm{F}=233.749$ & $<0.001$ \\
$\begin{array}{l}\text { Glutamine }(\mu M) \\
\text { Control }\end{array}$ & 767.876 & {$[751.039-784.713]$} & & \\
$\quad$ DRE & 781.459 & {$[759.757-803.162]$} & $\mathrm{F}=0.955$ & 0.33 \\
$\begin{array}{l}\text { Glutamate }(\mu M) \\
\text { Control }\end{array}$ & 113.167 & {$[108.175-118.16]$} & & \\
DSE & 121.886 & {$[113.487-130.284]$} & $\mathrm{F}=3.12$ & 0.079 \\
$\begin{array}{l}\text { Glutamine }(\mu M) \\
\text { Control }\end{array}$ & 766.568 & {$[749.486-783.649]$} & & \\
DSE & 859.253 & {$[830.517-887.989]$} & F=30.118 & $<0.001$ \\
\hline \multicolumn{4}{c}{ DRE - drug-resistant epilepsy, DSE - drug-response epilepsy, CI - confidence interval } \\
\hline
\end{tabular}


Table 3 - Univariate and multivariate logistic regression analyses of variables able to discriminate between DRE patients and controls.

\begin{tabular}{lcccc}
\hline Variables & Crude OR [95\% CI] & $P$-value & Adjusted OR [95\% CI] & $P$-value \\
\hline Gender & Ref. & & & \\
Female & $1.129[0.64-1.991]$ & 0.675 & & \\
Male & $0.947[0.906-0.990]$ & 0.017 & $0.963[0.899-1.03]$ & 0.273 \\
Age & $0.989[0.911-1.073]$ & 0.786 & & \\
BMI & $1.071[1.051-1.093]$ & $<0.001$ & $1.071[1.051-1.093]$ & $<0.001$ \\
Glutamate & $1.002[0.999-1.005]$ & 0.176 & & \\
Glutamine & DRE - drug-resistant epilepsy, OR - odds ratio, CI - confidence interval & \\
\hline \multicolumn{5}{c}{}
\end{tabular}

an increased extracellular glutamate level in the epileptogenic hippocampus in complex partial epilepsy resistant to pharmacotherapy during seizures related with the non-epileptogenic hippocampus. ${ }^{4}$ Cavus et al, ${ }^{12}$ also compared interictal extracellular glutamate levels among various sites in patients with medically refractory epilepsy, and their results demonstrated that in the epileptogenic brain areas including the cortex and hippocampus and lesional cortical sites, the extracellular glutamate levels were higher than those in the non-epileptogenic cortex and hippocampus.

However, the plasma glutamine levels in the DRE patients were similar to those in controls. A previous study revealed that the glutamine level in the extracellular fluid of the brain was reduced by chronic electrographic seizures in a kainate-induced rat model of epilepsy. ${ }^{13}$ However, our finding is consistent with previous studies reporting that interictal levels of extracellular glutamine are stable in DRE patients across any of the cortical (nonepileptogenic, epileptogenic, non-localized, lesional) sites or hippocampal (non-epileptogenic, epileptogenic) sites. ${ }^{12,14}$ Given that most existing studies have found positive associations between blood and cerebrospinal fluid levels of glutamate and glutamine, we speculated the alterations in plasma glutamate and glutamine may also reflect changes in the brain. Our results support the hypothesis that glutamate dysregulation occurs in DRE and may contribute to ongoing seizures. They also suggest that currently available therapies do not effectively reduce aberrantly high extracellular glutamate levels in patients with DRE.

Changes in extracellular glutamate concentrations are considered as an output of neuron-astrocyte unit activity. ${ }^{15,16}$ Both neurons and astrocytes can release and take up glutamate, and both cell types are involved in regulating extracellular amounts of glutamate. ${ }^{17,18}$ Excitatory amino acid transporters (EAATs) localized in astrocytes and neurons catalyze glutamate metabolism and diminish extracellular glutamate. ${ }^{19}$ Glial EAAT1
(GLAST) and EAAT2 (GLT-1) are the most crucial of these transporters, since they are responsible for glutamate uptake throughout the brain. ${ }^{19}$ In patients with drug-resistant temporal lobe epilepsy, the hippocampal expression levels of glial EAAT1 (GLAST) and EAAT2 (GLT-1) were shown to be decreased. ${ }^{17}$ These long-term alterations in EAAT expression indicate that EAATs may play a role in plasma glutamate abnormalities in patients with DRE.

Glutamate in the synaptic cleft is first transported with astrocytic transporters and rapidly converted to glutamine by glutamine synthetase (GS) in astrocytes. Glutamine synthetase has been assumed to control the glutamate metabolism rate and play a key role in glutamate cycling. ${ }^{2}$ Reduced GS protein expression and activity were confirmed in the hippocampus of temporal lobe epilepsy patients with hippocampal sclerosis relative to patients without sclerosis and in non-epileptic control subjects. ${ }^{16}$ Moreover, GS deficiency has been shown to be more pronounced in the hippocampus with astroglial proliferation. ${ }^{20}$ Glutamine synthetase deficiency inhibits the astrocytic glutamate-glutamine cycle and results in a higher load of extracellular glutamate. However, we found no difference in plasma glutamine levels between the DRE patients and controls. These data support the hypothesis that glial proliferation or another mechanism might compensate for GS deficiency to maintain steady extracellular glutamine levels. ${ }^{21}$

Alterations of plasma glutamate and glutamine level in DSE patients. Notably, the plasma glutamate levels in the DSE patients were similar to those in the healthy controls, whereas the plasma glutamine levels in the DSE patients were significantly elevated compared with those in controls. Glutamine is synthesized and released by astrocytes, and its elevation may be due to increased astrocytic GS function. Previous studies have reported increased GS function in the latent phase and during epilepsy progression in animal models. ${ }^{21,22}$ Therefore, the unchanged plasma glutamate levels and considerably 
higher glutamine levels in DSE may be attributable to a transiently promoted glutamate-glutamine cycle by increased GS function and to current AED therapies that could indirectly reduce aberrantly high extracellular glutamate. Collectively, the results of the present study revealed that plasma glutamate/glutamine alterations in patients with DRE and DSE may be consistent with dynamically changing GS function in the different states of epilepsy. ${ }^{22}$

Factors that may affect the plasma glutamate level in DRE patients. Our analysis also showed that male gender was associated with an increased plasma glutamate concentration in the DRE patients, which is in agreement with a previous study that demonstrated that the blood glutamate level is inversely correlated with

Table 4 - Univariate logistic regression analysis of factors able to discriminate between DSE patients and controls.

\begin{tabular}{lcc}
\hline Variables & Crude OR [95\% CI] & $P$-value \\
\hline Gender & Ref. & \\
$\quad$ Female & $0.929[0.471-1.829]$ & 0.83 \\
$\quad$ Male & $0.974[0.928-1.022]$ & 0.282 \\
Age & $0.982[0.873-1.106]$ & 0.769 \\
BMI & $1.01[0.999-1.021]$ & 0.083 \\
Glutamate & $1.009[1.005-1.012]$ & $<0.001$ \\
Glutamine &
\end{tabular}

DSE - drug-response epilepsy, OR - odds ratio, CI - confidence interval

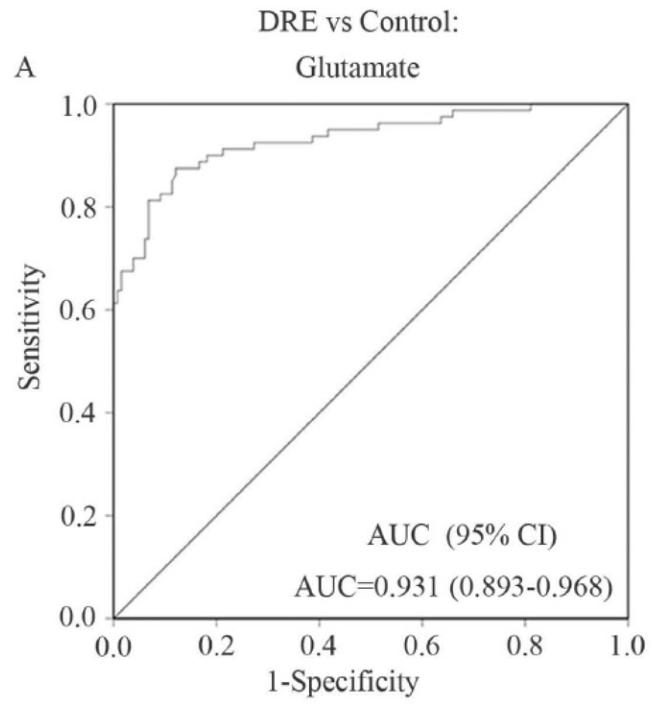

the levels of blood estrogen and progesterone, which were involved in the decrease in blood glutamate. ${ }^{23}$ Additionally, ordinal logistic regression analysis also showed that an elevated BMI was associated with a higher plasma glutamate concentration. Circulating glutamate was increased in patients with obesity resulting from impaired metabolism of glutamate in the adipocytes of individuals with obesity. ${ }^{24}$

Although gender and BMI were shown to impact the plasma glutamate concentration, no significant differences in these variables were found between the DRE patients and the controls, indicating that the variation in these variables did not confound our results. Neither age nor duration of epilepsy was linked with variation in the plasma glutamate concentration in the DRE patients. Our results also showed that seizure frequency was not correlated with an altered plasma glutamate concentration in the DRE patients, which is consistent with a preceding study revealing that high-frequency electrical stimulation (HFS) in an animal model with pharmacoresistant seizures did not alter extracellular glutamate levels in the hippocampus but did induce transient release of glutamate in the hippocampus of normal animals..$^{25,26}$ These data indicate that epilepsy alters the mechanisms underlying the HFS-induced increase of extracellular glutamate in the normal brain.

Additionally, polytherapy with AEDs was not

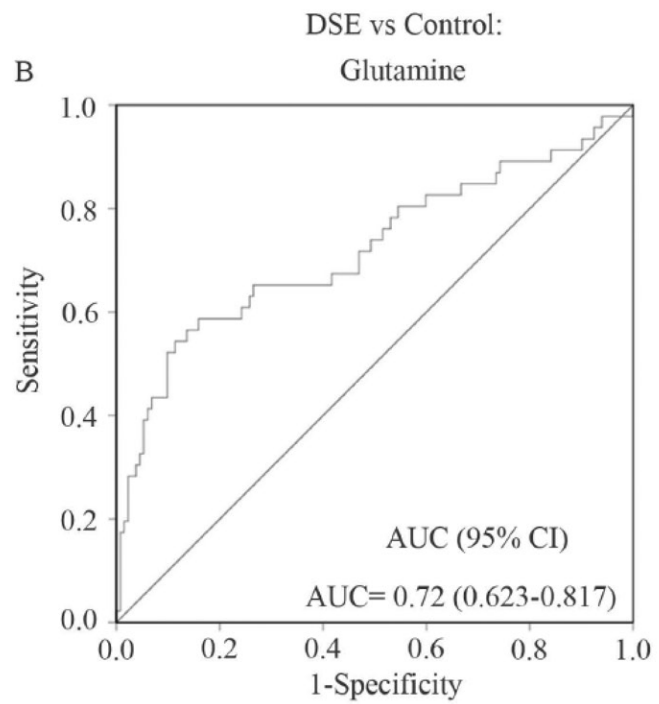

Figure 1 - Receiver operating characteristic (ROC) curve analysis of the ability of the plasma glutamate level to distinguish drugresistant epilepsy (DRE) patients from healthy controls (A). Receiver operating characteristic curve analysis of the ability of the plasma glutamine level to distinguish drug-responsive epilepsy (DSE) patients from healthy controls (B). 
Table 5 - Univariate and multivariate ordinal logistic regression analyses for association between tertile glutamate levels and factors in the DRE group.

\begin{tabular}{|c|c|c|c|c|}
\hline Variable & Crude OR [95\% CI] & $P$-value & Adjusted OR [95\% CI] & $P$-value \\
\hline \multicolumn{5}{|l|}{ Gender } \\
\hline $\begin{array}{l}\text { Female } \\
\text { Male }\end{array}$ & $\begin{array}{c}1 \\
6.526[2.571-16.567]\end{array}$ & $<0.001$ & $\begin{array}{c}1 \\
6.021[2.344-15.468]\end{array}$ & $<0.001$ \\
\hline Age & $0.987[0.932-1.046]$ & 0.665 & & \\
\hline BMI & $1.124[1.024-1.233]$ & 0.014 & $1.109[1.006-1.222]$ & 0.037 \\
\hline Duration of epilepsy & $1.011[0.966-1.058]$ & 0.645 & & \\
\hline \multicolumn{5}{|l|}{ Seizure frequency } \\
\hline $\begin{array}{l}\text { Daily } \\
\text { Weekly } \\
\text { Monthly }\end{array}$ & $\begin{array}{c}1 \\
0.874[0.304-2.514] \\
1.253[0.493-3.187]\end{array}$ & $\begin{array}{l}0.803 \\
0.635\end{array}$ & & \\
\hline \multicolumn{5}{|c|}{ AED utilization profile at the last clinic visit } \\
\hline $\begin{array}{l}\text { Monotherapy } \\
\text { Polytherapy }\end{array}$ & $\begin{array}{c}1 \\
0.967[0.325-2.879] \\
\end{array}$ & 0.951 & & \\
\hline
\end{tabular}

correlated with variation in the plasma glutamate level in DRE patients. Thus, there is still no evidence of any direct effects of AEDs on the glutamate level. ${ }^{27}$ Anti-epileptic drugs such as PHT, CBZ, OXZ, TPM, and VPA, which block repetitive activation of sodium channels, are likely to indirectly decrease glutamate release. ${ }^{28}$ However, cerebral glutamate concentrations were not changed after treatment with VPA, TPM or LEV in previous longitudinal studies. ${ }^{28-30}$ In the current study, the median plasma glutamate level was higher in the DRE patients than in the controls. This difference was probably not due to the effect of AEDs in our study, because the AEDs used in our study would most likely reduce the extracellular glutamate levels.

Limitations. First, the sample size was small, and the symptomatic focal epilepsy patients studied might not represent the patient population with epilepsy as a whole. Therefore, large-scale independent studies of patients with epilepsy of different etiologies will be required to validate our findings. Furthermore, in the current study, the disease duration among the DRE patients was relatively long. To minimize the impact of disease duration, studies of drug-resistant patients with a relatively short disease duration are needed to confirm our observations. Finally, we investigated plasma glutamate and glutamine levels in this study, and to confirm the present findings, D-glutamate as well as other NMDAR-co-agonists including D-serine and $\mathrm{D}$-alanine should also be investigated in future studies.

In conclusion, we observed a significant increase in plasma glutamate levels in patients with drug-resistant symptomatic focal epilepsy compared with the levels in controls and a significant increase in plasma glutamine levels in patients with drug-responsive symptomatic focal epilepsy compared with controls. Our results revealed that plasma glutamate levels could robustly discriminate the DRE patients from controls. Our results indicate that plasma glutamate levels can be used in the identification of patients at risk for symptomatic focal DRE. This will facilitate the timely administration of non-pharmacological interventions such as vagal nerve stimulation and epilepsy surgery to improve the quality of life of these patients and decrease the negative side effects of various AEDs.

Acknowledgment. The authors gratefully acknowledge Medjaden Inc. for English language editing.

\section{References}

1. Farrelly AM, Vlachou S, Grintzalis K. Efficacy of phytocannabinoids in epilepsy treatment: novel approaches and recent advances. Int J Environ Res Public Health 2021; 18 : 3993.

2. Leke R, Schousboe A. The glutamine transporters and their role in the glutamate/GABA-glutamine cycle. Adv Neurobiol 2016; 13: 223-257.

3. Robinson MB, Jackson JG. Astroglial glutamate transporters coordinate excitatory signaling and brain energetics. Neurochem Int 2016; 98: 56-71.

4. Alcoreza OB, Patel DC, Tewari BP, Sontheimer H. Dysregulation of ambient glutamate and glutamate receptors in epilepsy: an astrocytic perspective. Front Neurol 2021; 12: 652159.

5. Peterson AR, Binder DK. Astrocyte glutamate uptake and signaling as novel targets for antiepileptogenic therapy. Front Neurol 2020; 11: 1006.

6. Price JB, Rusheen AE, Barath AS, Rojas Cabrera JM, Shin $\mathrm{H}$, Chang SY, et al. Clinical applications of neurochemical and electrophysiological measurements for closed-loop neurostimulation. Neurosurg Focus 2020; 49: E6. 
7. Hashimoto K, Bruno D, Nierenberg J, Marmar CR, Zetterberg $\mathrm{H}$, Blennow $\mathrm{K}$, et al. Abnormality in glutamine-glutamate cycle in the cerebrospinal fluid of cognitively intact elderly individuals with major depressive disorder: a 3-year follow-up study. Transl Psychiatry 2016; 6: e744.

8. Madeira C, Vargas-Lopes C, Brandão CO, Reis T, Laks J, Panizzutti R, et al. Elevated glutamate and glutamine levels in the cerebrospinal fluid of patients with probable alzheimer's disease and depression. Front Psychiatry 2018; 9: 561.

9. Kwan P, Arzimanoglou A, Berg AT, Brodie MJ, Allen Hauser W, Mathern G, et al. Definition of drug resistant epilepsy: consensus proposal by the ad hoc task force of the ILAE commission on therapeutic strategies. Epilepsia 2010; 51: 1069-1077.

10. Siddiqui A, Kerb R, Weale ME, Brinkmann U, Smith A, Goldstein DB, et al. Association of multidrug resistance in epilepsy with a polymorphism in the drug-transporter gene ABCB1. N Engl J Med 2003; 348: 1442-1448.

11. Kumar R, Indrayan A. Receiver operating characteristic (ROC) curve for medical researchers. Indian Pediatr 2011; 48: 277-287.

12. Çavuş I, Romanyshyn JC, Kennard JT, Farooque P, Williamson A, Eid $\mathrm{T}$, et al. Elevated basal glutamate and unchanged glutamine and GABA in refractory epilepsy: microdialysis study of 79 patients at the yale epilepsy surgery program. Ann Neurol 2016; 80: 35-45.

13. Kanamori K, Ross BD. Chronic electrographic seizure reduces glutamine and elevates glutamate in the extracellular fluid of rat brain. Brain Res 2011; 1371: 180-191.

14. Cavus I, Kasoff WS, Cassaday MP, Jacob R, Gueorguieva R, Sherwin RS, et al. Extracellular metabolites in the cortex and hippocampus of epileptic patients. Ann Neurol 2005; 57: 226-235.

15. Zavvari F, Modarres Mousavi SM, Ejlali M, Barfi S, Karimzadeh F. Glutamate Signaling pathway in absence epilepsy: possible role of ionotropic AMPA glutamate receptor type 1 subunit. Iran J Pharm Res 2020; 19: 410-418.

16. Sandhu MRS, Gruenbaum BF, Gruenbaum SE, Dhaher R, Deshpande K, Funaro MC, et al. Astroglial glutamine synthetase and the pathogenesis of mesial temporal lobe epilepsy. Front Neurol 2021; 12: 665334.

17. Todd AC, Hardingham GE. The regulation of astrocytic glutamate transporters in health and neurodegenerative diseases. Int J Mol Sci 2020; 21: 9607.

18. Passlick S, Rose CR, Petzold GC, Henneberger C. Disruption of glutamate transport and homeostasis by acute metabolic stress. Front Cell Neurosci 2021; 15: 637784.

19. Alleva C, Machtens JP, Kortzak D, Weyand I, Fahlke C. Molecular basis of coupled transport and anion conduction in excitatory amino acid transporters. Neurochem Res 2021 Feb.
20. Wang H, Huang Y, Coman D, Munbodh R, Dhaher R, Zaveri HP, et al. Network evolution in mesial temporal lobe epilepsy revealed by diffusion tensor imaging. Epilepsia 2017; 58: 824-834.

21. Eid T, Lee TW, Patrylo P, Zaveri HP. Astrocytes and glutamine synthetase in epileptogenesis. J Neurosci Res 2019; 97: 1345-1362.

22. Sun HL, Zhu W, Zhang YR, Pan XH, Zhang JR, Chen $\mathrm{XM}$, et al. Altered glutamate metabolism contributes to antiepileptogenic effects in the progression from focal seizure to generalized seizure by low-frequency stimulation in the ventral hippocampus. Epilepsy Behav 2017; 68: 1-7.

23. Rossetti MF, Cambiasso MJ, Holschbach MA, Cabrera R. Oestrogens and progestagens: synthesis and action in the brain. J Neuroendocrinol 2016; 28.

24. Maltais-Payette I, Boulet MM, Prehn C, Adamski J, Tchernof A. Circulating glutamate concentration as a biomarker of visceral obesity and associated metabolic alterations. Nutr Metab (Lond) 2018; 15: 78.

25. Luna-Munguia H, Zestos AG, Gliske SV, Kennedy RT, Stacey WC. Chemical biomarkers of epileptogenesis and ictogenesis in experimental epilepsy. Neurobiol Dis 2019; 121: 177-186.

26. Luna-Munguía H, Meneses A, Peña-Ortega F, Gaona A, Rocha L. Effects of hippocampal high-frequency electrical stimulation in memory formation and their association with amino acid tissue content and release in normal rats. Hippocampus 2012; 22: 98-105.

27. Rogawski MA, Löscher W, Rho JM. Mechanisms of action of antiseizure drugs and the ketogenic diet. Cold Spring Harb Perspect Med 2016; 6: a022780.

28. van Veenendaal TM, IJff DM, Aldenkamp AP, Lazeron RHC, Puts NAJ, Edden RAE, et al. Glutamate concentrations vary with antiepileptic drug use and mental slowing. Epilepsy Behav 2016; 64: 200-205.

29. Leite CDC, Valente KDR, Fiore LA, Otaduy MCG. Proton spectroscopy of the thalamus in a homogeneous sample of patients with easy-to-control juvenile myoclonic epilepsy. Radiol Bras 2017; 50: 279-284.

30. Pichardo Macías LA, Ramírez Mendiola BA, Contreras García IJ, Zamudio Hernández SR, Chávez Pacheco JL, Sánchez Huerta KB, et al. Effect of levetiracetam on extracellular amino acid levels in the dorsal hippocampus of rats with temporal lobe epilepsy. Epilepsy Res 2018; 140: 111-119. 